\title{
Call for infection control to stem MERS
}

$\mathrm{T}$ he World Health Organization (WHO) affirmed that the accelerating outbreak of Middle East respiratory syndrome (MERS) is not an international emergency, but delivered a strong message to Saudi Arabia to improve its "suboptimal" infection control and complete needed surveillance studies.

The recent acceleration of cases (235 of the 496 cases confirmed to date have occurred since Apr. 11) is due mainly to hospital-based outbreaks and an increase in cases in the community, said Dr. Keiji Fukuda, assistant director-general for health security during a virtual press conference from Geneva, Switzerland, May 13. He said there is no evidence of sustained human-to-human transmission of MERS in community settings that would cross the threshold for an international emergency.

A WHO mission that visited hospitals in Saudi Arabia Apr. 28 to May 5 found that control measures were "not up to standard." The mission found overcrowded waiting rooms in emergency departments and problems with basic infection control measures such as handwashing, gloves and masks, said Fukuda. The WHO's emergency committee on MERS' top recommendation was for better infection prevention and control in the main host MERS countries.

Community surveillance is also a problem; case-control and serologic surveillance studies recommended previously by the committee have not been completed. As a result, there are key gaps in information and the sources of

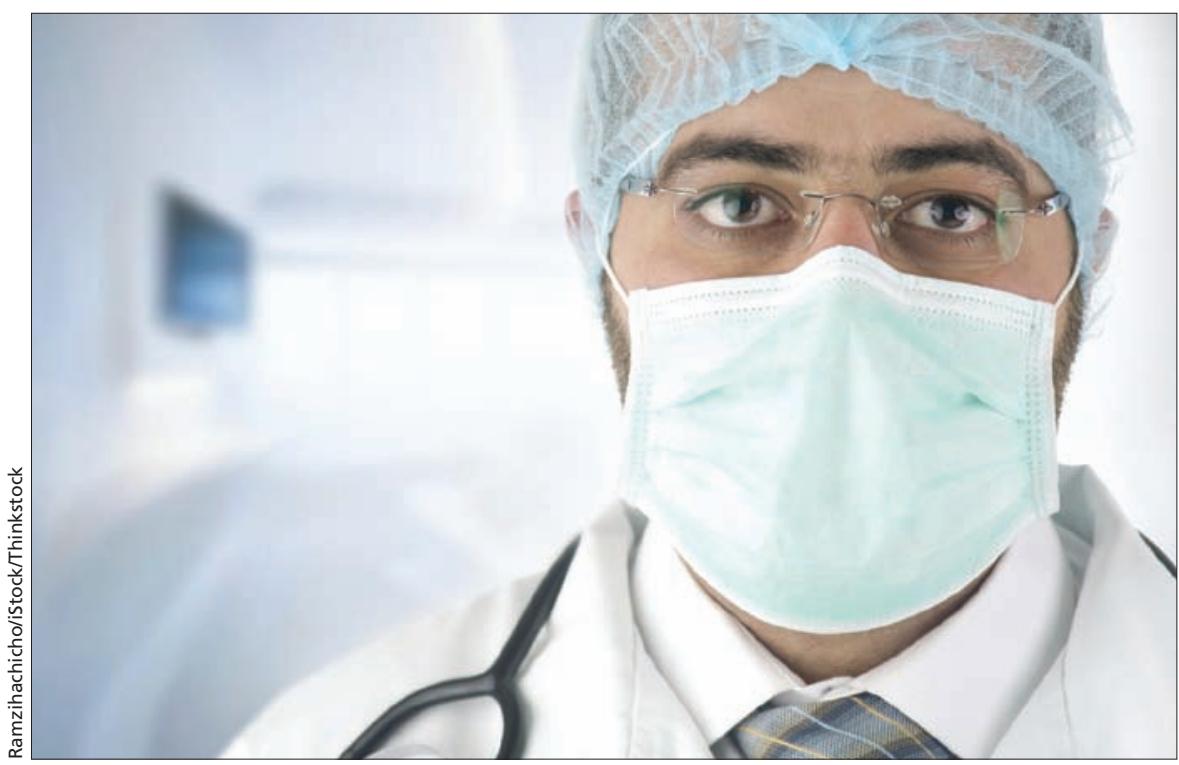

A WHO mission to Saudi Arabia found basic infection control measures, such as wearing masks, were "not up to standard."

infection in the community are still unclear, said Fukuda. WHO has been helping Saudi Arabia with study protocols and other support.

The cause of a recent uptick in community cases is also unclear. It may be due to a seasonal or general increase in MERS in camels, which are believed to be the source of the infection. Or it may be attributed to better surveillance and detection, or to increased transmissibility from human-to-human, said Fukuda. The final possibility is most worrying, said Fukuda, but he noted that samples of the MERS virus recently exported to other countries by travellers are genetically similar to earlier viral samples sequenced, meaning that the virus has mutated very little.
Because of the evolving MERS picture, the 16-member emergency committee, which includes a Canadian representative, plans to meet again in the next few weeks for its sixth meeting. It has followed the outbreak since July 2013. Under the International Health Regulations, which took effect in 2007 , WHO convenes an emergency committee during outbreaks to recommend measures to WHO and national public health organizations. Emergency committees have declared a "public health emergency of international concern" twice, for the H1N1 influenza pandemic in 2009 and for the international spread of polio this year. - Carolyn Brown, Ottawa, Ont.

CMAJ 2014. DOI:10.1503/cmaj.109-4806 